\title{
O PRÊMIO NOBEL DE FÍSICA (PNF) DE $2008^{+*}$
}

José Maria Filardo Bassalo

Fundação Minerva

Belém - PA

\section{Resumo}

Neste artigo, trataremos do Prêmio Nobel de Física de 2008, concedido aos fisicos japoneses Yoichiro Nambu, Makoto Kobayashi e Toshihide Maskawa, por haverem descoberto mecanismos envolvendo simetrias nas interações fortes (quiral), por Nambu, e nas interações fracas (carga-paridade), por Kobayashi e Maskawa.

Palavras-chave: Prêmio Nobel de Física de 2008; Nambu; Kobayashi; Maskawa; Simetrias: quiral e carga-paridade.

\begin{abstract}
In this article we will talk about the Nobel Prize in Physics 2008, granted to the Japanese physicists Yoichiro Nambu, Makoto Kobayashi and Toshihide Maskawa, for their discovery of the mechanisms involving strong interactions symmetries (quiral), by Nambu, and in weak interactions (charge-parity), by Kobayashi and Maskawa.
\end{abstract}

Keywords: 2008` Nobel Prize of Physics; Nambu; Kobayashi; Maskawa; Symmetries: quiral and charge-parity.

O PNF de 2008 foi concedido aos físicos japoneses Yoichiro Nambu (n. 1921) (naturalizado norte-americano), Makoto Kobayashi (n. 1944) e Toshihide

\footnotetext{
${ }^{+}$Physics Nobel Prize (PNP) in 2008

* Recebido: dezembro de 2008. Aceito: março de 2009.
} 
Maskawa (n. 1940). Nambu descobriu uma nova maneira de realizar uma simetria das interações fortes: a simetria quiral. Por seu lado, Kobayashi e Maskawa propuseram mecanismos para produzir a violação da simetria carga-paridade (CP) em uma Teoria de Interações Fracas.

A simetria tem representado um papel importante no estudo da Física. Vejamos como isso aconteceu. Segundo Rosen e Freudlich : "A Natureza apresenta simetrias e conservações, cuja associação reflete leis da própria Natureza.” Dessa forma, o Homo Sapiens, ao formular modelos e teorias por intermédio daquela associação, as quais, consequentemente, traduziam aspectos das leis naturais, revelou que, tanto a simetria em si como a quebra de algum padrão de simetria, poderia servir de instrumento para obtenção de conhecimento .

Foi o químico e físico francês Pierre Curie (1859-1906; PNF, 1903) o primeiro a introduzir a importância da simetria no estudo dos fenômenos físicos, ao afirmar que "são as assimetrias que possibilitam os fenômenos". Para ele, uma exata simetria da Natureza não poderia ser detectada, já que todos os pontos do Universo seriam indistinguíveis, e a probabilidade da realização de experiências seria a mesma. Por outro lado, ao fazer a distinção clara entre vetores polares e axiais, Pierre Curie percebeu a importância da Teoria de Grupos ${ }^{3}$ no estudo dos fenômenos físicos. É oportuno esclarecer que, em Física, chama-se de simetria a toda transformação que leva um sistema físico a um outro que lhe seja equivalente, decorrendo daí uma invariância desse sistema. O conjunto de transformações de simetria forma um grupo. Observe-se que se denomina de vetor polar ao vetor que apresenta a direção independente do sistema de coordenadas; em caso contrário, ele se denomina de vetor axial ou pseudo-vetor. Por exemplo, os vetores posição $(\vec{r})$ e momento linear $(\overrightarrow{\mathrm{p}}=\mathrm{md} \overrightarrow{\mathrm{r}} / \mathrm{dt}=\mathrm{m} \overrightarrow{\mathrm{v}})$ são vetores polares; o vetor momento angular $(\vec{L}=\vec{r} \times \vec{p})$ é um vetor axial.

Um dos primeiros grupos usados na Física foi o grupo de Galileu, que decorre dos trabalhos realizados pelo matemático e físico italiano Galileu Galilei (1564-1642), nos quais ele mostrou, em 1638, em seu famoso livro intitulado Diá-

${ }^{1}$ ROSEN; J.; Y. FREUNDLICH, Y. American Journal of Physics, n. 46, p. 1030, 1978.

2 FLEMING, H. Ciência e Filosofia, n. 1, p. 99, 1979.

3 Inventada pelo matemático francês Évariste Galois (1811-1832), em 1830/1831, como se pode ver em TATON, 2007. TATON, R. Dicionário de Biografias Científicas. Contraponto, 2007. v. II 
logos Concernentes a Duas Novas Ciências, que a velocidade (v) de um objeto em relação a um outro corpo em repouso é igual à velocidade (v') que ele tem em relação a um outro corpo que se desloca com velocidade constante $(\mathrm{V})$ em relação ao corpo parado, acrescida dessa última velocidade, isto é: $v=v^{\prime}+V$. Em linguagem atual, isso significa dizer que as leis da Mecânica são invariantes por uma transformação (grupo) de Galileu.

Muito embora as leis de conservação em Física já fossem conhecidas há muito tempo, como a conservação do momento linear , a conservação do momento angular ${ }^{5}$, e a conservação da energia mecânica ${ }^{6}$, sua relação com a simetria só foi formulada em $1918^{7}$ pela matemática alemã Amalie Emmy Noether (1882-1935), ao demonstrar que as constantes de movimento de um sistema físico, isto é, os seus invariantes, estão associadas com os grupos de simetria das transformações equivalentes. Por exemplo, quando o Lagrangeano (L), ou seja, a diferença entre as energias cinética $(\mathrm{T})$ e potencial $(\mathrm{V})-\mathrm{L}=\mathrm{T}-\mathrm{V}$, que determina as equações de movimento (por intermédio da equação de Euler-Lagrange) de um sistema físico apresentar simetria de translação no tempo, na posição, e apresentar, também, simetria de rotação no espaço, teremos, respectivamente, as Leis de Conservação da Energia (E), do Momento Linear (p) e do Momento Angular (L), o que significa dizer, portanto, que essas grandezas físicas são invariantes. Destaquese que essa demonstração ficou conhecida como o Teorema de Noether.

O estudo dos princípios de simetria e a aplicação da Teoria de Grupos aos sistemas de muitos-elétrons foi iniciado pelo físico húngaro norte-americano Eugene Paul Wigner (1902-1995; PNF, 1963), em $1926^{\circ}$. Com esse estudo, ele esclareceu o aforisma de Pierre Curie enunciado acima, observando que a descrição de um fenômeno físico depende de suas condições iniciais. Desse modo, é a assimetria das condições iniciais que permite determinar as simetrias das leis da Natureza. A separação entre as condições iniciais e as leis da Natureza surge, naturalmente, quando se representa um fenômeno natural por intermédio de uma equação

\footnotetext{
${ }^{4}$ Pelo matemático inglês John Wallis (1616-1738), em 1668.

${ }^{5}$ Pelo físico e matemático suíço Leonhard Euler (1707-1783), em 1751.

${ }^{6}$ Pelo físico e matemático francês Gustave-Gaspard Coriolis (1792-1843), em 1829.

${ }^{7}$ Königlichi Gesellschaft der Wissenschaften zu Göttingen Nachrichten, p. 37; 235, 1918.

${ }^{8}$ Zeitschrift fur Physik, v. 40, p. 492, 1926.
} 
diferencial, já que, para resolvê-la, é necessário conhecer as condições iniciais. Daí o grande sucesso dos formalismos diferenciais no estudo dos fenômenos físicos.

Por outro lado, ao estudar as Leis de Conservação na Mecânica Quântica, Wigner observou, em $1927^{9}$, que tais leis são associadas à existência de operadores unitários $\mathrm{P}$ [operador paridade (reflexão)], de autovalores \pm 1 , que comutam com o operador Hamiltoniano $\mathrm{H}(\mathrm{H}=\mathrm{T}+\mathrm{V})$. Registre-se que Wigner continuou a produzir trabalhos relacionados com a aplicação da Teoria de Grupos na Mecânica Quântica, os quais reuniu em seu célebre livro intitulado Gruppentheorie und ihre Anwendung auf die Quantenmechanik der Atomspektern, publicado em $1931^{10}$. Nesse livro, ele propôs a Lei de Conservação da Paridade P, segundo a qual nenhuma experiência seria capaz de determinar, de maneira unívoca, a direita ou a esquerda. Observe-se que a violação dessa lei foi proposta teoricamente, em 1956, pelos físicos sino-norte-americanos Tsung-Dao Lee (n. 1926; PNF, 1957) e Chen Ning Yang (n. 1922; PNF, 1927) e confirmada em uma experiência, em 1957, sob a liderança da física sino-norte-americana Madame Chien-Shiung Wu (1912-1997) respectivamente. Registre-se, também, que, em $1932^{11}$, Wigner estudou a reflexão no tempo - o operador inversão temporal $\mathrm{T}$ - nos fenômenos físicos.

É oportuno destacar que a aplicação da Teoria de Grupos à Mecânica Quântica também foi estudada pelo físico e matemático alemão Hermann Klaus Hugo Weyl (1885-1955), em seu famoso livro intitulado Gruppentheorie und Quantenmechanik, publicado em Leipzig, em 1928. Nesse livro, Weyl mostrou que os resultados da Mecânica Quântica (desenvolvida a partir de 1925) aplicados ao átomo de hidrogênio $(\mathrm{H})$, poderiam ser simplesmente entendidos por intermédio da Teoria de Grupos, uma vez que os números quânticos, que aparecem na Teoria Quântica desse átomo, são índices que caracterizam as representações do Grupo de Rotações.

A descoberta de novas partículas elementares fez com que novas simetrias e correspondentes leis de conservação fossem então propostas. Com efeito, a descoberta do nêutron como constituinte do núcleo atômico, em 1932, levou à propo-

${ }^{9}$ Zeitschrift fur Physik, v. 43, p. 624, 1927.

${ }^{10}$ Group Theory and its Applications to the Quantum Mechanics. Academic Press, 1959.

\footnotetext{
11

Akademie der Wissenschaften zu Göttingen Nachrichten, Mathematischphysikalische Klasse, p. 546, 1932.
} 
sição da simetria do spin isotópico ou isospin (I) [simetria do $\mathrm{SU}(2)$ ] para poder explicar a estabilidade desse núcleo. Por sua vez, a descoberta dos píons $(\pi)$, das partículas estranhas (p.e.: $\Lambda, \mathrm{K}, \Sigma, \Xi$ ), a partir de 1947 , e das ressonâncias (p.e: $\Delta, \Sigma^{*}, \Xi^{*}, \Omega$ ), a partir de 1952 , levou a proposição de uma outra simetria - a estranheza (S). Registre-se que essas simetrias não são conservadas em todas as interações físicas que ocorrem na Natureza.

Excetuando-se a simetria de spin isotópico (que é uma simetria interna) as outras simetrias que vimos até aqui (translação, rotação e reflexão) são simetrias geométricas, pois dependem apenas da posição geométrica de dois observadores que analisam o mesmo fenômeno, ou equivalentemente, o mesmo observador examinando um determinado fenômeno de duas posições geométricas diferentes. No entanto, existem outras simetrias que não dependem da posição geométrica de quem examina um determinado fenômeno físico. Dentre essas simetrias, duas são muito importantes no estudo da Física. A primeira delas, a inversão temporal $\mathrm{T}$, estudada por Wigner, conforme vimos, significa que, se um certo movimento de um sistema físico é possível, seu reverso temporal também o será. Em linguagem matemática, essa simetria ocorre quando as leis físicas que descrevem um determinado fenômeno físico não se alteram quando se troca o tempo $t$ por $-t$. Note-se que a Física Clássica, representada, basicamente, pela equação de Newton e pelas equações de Maxwell, são invariantes pelo operador T, já que tais equações envolvem derivadas (total e parcial) segundas envolvendo o tempo $(t)$. Ora, como essa inversão temporal $\mathrm{T}$ é preservada na Física Clássica, ela foi estendida à Física Contemporânea, principalmente à Física das Partículas Elementares.

A outra simetria não-geométrica importante no estudo dos fenômenos físicos é a denominada conjugação de carga $C$, segundo a qual uma experiência física permanece invariante quando se troca a partícula por sua antipartícula correspondente. Registre-se que as antipartículas (com carga elétrica de sinal contrário das partículas correspondentes) foram previstas pela equação de Dirac, de 1928.

A invariância das simetrias $\mathrm{CP}$ e T, que decorre da invariância de Lorentz em uma Teoria de Campo Local, foi estudada pelo físico norte-americano Julian Seymour Schwinger (1918-1994; PNF, 1965), em $1953^{12}$ e $1954^{13}$ e, também, pelo

\footnotetext{
${ }^{12}$ Physical Review, v. 91, p. 713; 728, 1953; v. 92, p. 1283, 1953. 13

Physical Review, v. 93; v. 94, p. 615, 1362, 1954.
} 
físico alemão Gerhart Luders (1920-1995), em $1954^{14}$. Registre-se que antes, em $1952^{15}$, os físicos, o italiano Gian Carlo Wick (1909-1992), o norte-americano Arthur Strong Wightman (n. 1922) e Wigner, introduziram a simetria CP para estudar as reações envolvendo as partículas elementares.

Em $1957^{16}$, Luders demonstrou o famoso Teorema CPT, segundo o qual os observáveis em Física são invariantes por uma transformação combinada, em qualquer ordem, das operações C, P e T. Ainda segundo esse Teorema, toda partícula possui uma antipartícula associada de mesma massa, mesma vida-média e de mesmo momento magnético da partícula correspondente, conforme foi demonstrado, ainda em 1957, em trabalhos independentes ${ }^{17}$ realizados pelos físicos norteamericanos de origem chinesa Tsung-Dao Lee (n. 1926; PNF, 1957), Reinhard Oehme (n. 1928) e Chen Ning Yang (n. 1925; PNF, 1957), também de origem chinesa, e, independentemente, pelos físicos russos Boris Lazarevich Ioffe (n. 1926), Lev Borisovich Okun (n. 1929) e A. P. Rudik ${ }^{18}$.

Ainda no trabalho de Lee, Oehme e Yang, eles discutiram a violação da simetria CP nas interações fracas. Muito embora, a comprovação experimental da existência de dois neutrinos (um associado ao elétron e outro associado ao múon), observada em 1962 e 1964, mostrasse que a interação fraca violasse, independentemente, as simetrias C e $\mathrm{P}$, porém, a simetria CP permanecia inviolável, assim como a simetria $\mathrm{T}$, por causa da inviolabilidade da simetria CPT. Contudo, a violação da CP foi comprovada, em 1964, em experiências independentes realizadas pelos físicos: os norte-americanos James H. Christenson, James Watson Cronin (n. 1921; PNF, 1980), Val Logsdon Fitch (n. 1923; PNF, 1980) e o francês René Turlay (n. 1932) ${ }^{19}$, e A. Abashian, R. J. Abrams, D. W. Carpenter, G. P. Fisher, B. M. K. Mefkens e J. H. Smith ${ }^{20}$. Nessas experiências, observaram a violação da sime-

${ }^{4}$ Matematisk-Fysiske Meddelelser Köngelige Danske Videnskabernes Selskab, v. 28, p. $1,1954$.

15

Physical Review, v. 88, p. 101, 1954.

Annals of Physics, v. 2, p. 1, 1957.

17

Physical Review, v. 106, p. 340, 1957.

18

Soviet Physics - JETP, v. 5, p. 328, 1957.

19

Physical Review Letters, v. 13, p. 138, 1964.

20

Physical Review Letters, v. 13, p. 243, 1964.

Bassalo, J. M. F. 
tria (ou "invariância") CP (troca de carga elétrica e inversão da paridade) do sistema $\mathrm{K}^{0}-\overline{\mathrm{K}}^{0}$. Note-se que, ainda em 1964, em trabalhos distintos realizados pelos físicos norte-americanos Lincoln Wolfenstein ${ }^{21}$ e Robert Green Sachs (1916$1999)^{22}$, eles propuseram uma nova interação na Natureza, a superfraca, para explicar aquela violação. Também em 1964, em trabalhos independentes de John Stuart Bell (1928-1990) e J. K. Perring ${ }^{23}$, e de J. Bernstein, Nicola Cabibbo (n.1935) e Lee ${ }^{24}$, eles sugeriram que a violação da simetria CP poderia ser devida a um campo vetorial de longo alcance e de origem cosmológica. (Registre-se que a explicação dada por Kobayashi e Maskawa sobre a violação da CP, conforme veremos mais adiante, descartou definitivamente, quer a existência da interação superfraca, quer a existência do campo vetorial cósmico.) Em $1965^{25}$, Bernstein, Feinberg e Lee observaram uma possível violação da simetria CP nas interações eletromagnéticas envolvendo hádrons.

Antes de falarmos dos trabalhos dos Nobelistas, façamos um pequeno estudo sobre outras simetrias. Com efeito, até aqui, falamos apenas das simetrias relacionadas com fenômenos físicos envolvendo as partículas elementares, no que se relaciona com a sua produção. No entanto, o estudo da simetria em Física é básico, pois ela é quem dita a interação. De um modo geral, uma interação física pode apresentar dois tipos de simetria: global e local. Na simetria global, a transformação que caracteriza a mesma é aplicada uniformemente a todos os pontos do espaço; na simetria local, cada ponto é transformado independentemente. Por exemplo, tomemos um balão e marquemos os seus meridianos e paralelos. Se girarmos esse balão em torno de um de seus diâmetros, a nova posição será simétrica à primeira, pois o balão mantém a mesma forma. Nesse caso, temos simetria global porque as posições de todos os pontos sobre o balão sofrem um mesmo deslocamento angular. Por outro lado, a simetria local requer que o balão mantenha a mesma forma, ainda que seus pontos sejam movidos independentemente, o que

\footnotetext{
${ }^{21}$ Physical Review Letters, v. 13, p. 286, 1964.

${ }^{22}$ Physical Review Letters, v. 13, p. 562, 1964.

23

Physical Review Letters, v. 13, p. 348, 1964.

24

Physics Letters, v. 12, p. 146, 1964.

25

Physical Review B, v. 139, p. 1650.
} 
provocará uma deformação nos meridianos e nos paralelos, em consequência da aplicação de forças nos diversos pontos do balão ${ }^{26}$.

Tendo em vista essas simetrias, vejamos o que ocorre com as interações. Se uma determinada interação é invariante por uma simetria global, e se exigirmos que a mesma seja invariante por uma simetria local, é necessário que se introduza na interação considerada novos campos (novas "forças") - chamados campos de gauge ("calibre") -, campos esses que vêm associados a partículas. É importante registrar que as teorias de campos de gauge não-abeliana na Física foram introduzidas desde $1954^{27}$ a partir do trabalho de Yang e do físico norteamericano Robert Lawrence Mills (n. 1927). É ainda oportuno registrar que uma teoria semelhante a essa foi apresentada pelo físico inglês Robert Shaw (n.1929), em sua Tese de Doutoramento, defendida em 1955, no Imperial College, na Inglaterra, sob a orientação do físico paquistanês Abdus Salam (1926-1996; PNF, 1979). Registre-se, também, que a Teoria de Yang-Mills-Shaw (TYMS) é uma extensão da simetria global de isospin para uma simetria local, extensão essa que induz a introdução de campos de gauge associados a partículas bosônicas (spin inteiro) e sem massa. Contudo, por não ser renormalizável para bósons massivos, essa TYMS não poderia descrever as interações fracas, pois estas são mediadas por partículas massivas, os bósons vetoriais intermediários: $W^{ \pm}, Z^{0}$. Essa renormalização foi realizada nos trabalhos que levaram à unificação das interações eletromagnética e fraca, a hoje conhecida interação eletrofraca, regida pelo grupo $\mathrm{SU}(2)_{\mathrm{L}} \otimes \mathrm{U}(1)_{\mathrm{Y}}{ }^{28}$.

Vejamos alguns exemplos sobre as simetrias vistas acima. A interação eletromagnética apresenta uma simetria global na fase do campo eletromagnético. Essa simetria é denominada de U (1) e está associada à conservação da carga elétrica. Porém, se quisermos que os observáveis envolvidos nessa interação (energia, por exemplo) permaneçam invariáveis quando a fase do campo $\{\exp [\mathrm{i} \alpha(\vec{r})]\}$ sofre um deslocamento em todos os seus pontos, teremos de introduzir um campo de gauge associado a uma partícula de spin 1 e sem massa, denominada fóton $(\gamma)$. Um segundo exemplo de teorias de gauge mediadas por bósons não-massivos

${ }^{26}$ FREEDMAN, D. Z; NIEUWENHUIZEN, P. V. Scientific American, v. 238, p. 126, Feb. 1978.

${ }^{27}$ Physical Review, v. 96, p. 191, 1954.

${ }^{28}$ Sobre o significado desses grupos, ver: BASSALO, J. M. F.; CATTANI, M. S. D. Teoria de Grupos. Livraria da Física, 2008. 
relaciona-se com a interação gravitacional. Esta pode ser entendida como consequência de uma invariância por uma transformação local (não-interna, isto é, envolvendo apenas translação e rotação no espaço-tempo) proposta pelo matemático francês Jules Henri Poincaré (1854-1912), em 1905, em carta que escreveu ao físico holandês Hendrik Antoon Lorentz (1853-1928; PNF, 1902). Observe-se que ao campo de gauge correspondente a essa invariância está associada uma partícula de spin 2 e sem massa, denominada gráviton, ainda não detectada ${ }^{29}$.

As duas interações analisadas acima são de longo alcance. No entanto, na Natureza existem mais dois tipos de interação - fraca e forte - que são, contudo, de curto alcance. A interação fraca é descrita por um campo de gauge do tipo Yang-Mills-Shaw, cuja simetria correspondente é a do grupo SU(2). Por seu lado, a interação forte é descrita pelo grupo local de gauge colorido $\mathrm{SU}_{\mathrm{C}}(3)$, cujas partículas mediadoras dessa interação são em número de 8 , de spin 1 , sem massa, com "carga de cor" e denominadas glúons. Note-se que a teoria que estuda a interação forte é conhecida como Cromodinâmica Quântica ("Quantum Chromodynamics" - QCD). A interação eletrofraca, conforme dissemos acima, é regida pelo grupo $\mathrm{SU}(2)_{\mathrm{L}} \otimes \mathrm{U}(1)_{\mathrm{Y}}$, é mediada pelas partículas: $\gamma, \mathrm{W}^{ \pm}, \mathrm{Z}^{0}$, e decorre da Teoria (TWS), desenvolvida pelo físico norte-americano Steven Weinberg (n. 1933; PNF, 1976), em 1967, e por Salam, em 1968. É interessante notar que a interação forte conserva a paridade (P), o isospin (I) e a estranheza (S); a interação fraca não conserva esses três números quânticos; e a interação eletromagnética viola a lei de conservação do isospin. É oportuno registrar que o grupo $\mathrm{SU}(2)_{\mathrm{L}}$ $\otimes U(1)_{Y}$ foi proposto em detalhe pelo físico norte-americano Sheldon Lee Glashow (n. 1932; PNF, 1979), em $1961^{30}$.

Agora, vejamos os trabalhos dos Nobelistas. Comecemos com Nambu. Um de seus primeiros trabalhos foi publicado em $1949^{31}$, no qual apresentou cálculos refinados do deslocamento ("shift") ou desvio Lamb. Em 1951", estudou, com a colaboração dos físicos japoneses Kakukiko Nishijima (n. 1926) e Y. Ya-

\footnotetext{
29

LOPES, J. L. Gauge Fields Theories: An Introduction. Pergamon Press, 1981; LEADER, E.; PREDAZZI, E. An introduction to Gauge Theories and the 'New Physics'. Cambridge University Press, 1983.

30

Nuclear Physics, v. 22, p. 519, 1961.

31

Progress in Theoretical Physics, v. 4, p. 82, 1949.

32

Progress in Theoretical Physics, v. 6, p. 615, 1951.
} 
maguchi, a produção associada das partículas estranhas $\left(\Lambda^{0}\right.$ e $\left.K^{0}\right)$. Ainda em $1951^{33}$, ele e Yamaguchi estudaram o espalhamento píon-núcleon $(\pi-\mathrm{N})$. Muito embora, ainda na década de 1950, Nambu tenha realizado outros trabalhos relacionados com o espalhamento de partículas, seu interesse pelo tema que o tornou Nobelista, qual seja, a quebra espontânea de simetria, só aconteceu no final dessa década. Inicialmente, Nambu tratou dessa quebra na supercondutividade. Vejamos como isso aconteceu.

A supercondutividade, descoberta em 1911 pelo físico holandês Heike Kamerlingh Onnes (1853-1926; PNF, 1913), indicava que, em temperaturas próximas do zero absoluto, alguns materiais apresentavam resistência elétrica praticamente nula. Durante muitos anos depois dessa descoberta, acreditou-se que, exceto pelo fato de apresentarem resistência quase nula, esses novos materiais, denominados posteriormente de supercondutores, possuíam as mesmas propriedades que os condutores normais. Somente em 1933, os físicos alemães Fritz Walther Meissner (1882-1974) e Robert Ochsenfeld (1901-1993) mostraram que o estado supercondutor é diamagnético, ao observarem que as linhas de indução de um campo magnético externo eram expulsas do interior de um cilindro longo de estanho (Sn), quando este era resfriado abaixo de sua temperatura crítica $T_{C}$ (temperatura em que ocorre a supercondutividade). Note-se que essa observação ficou conhecida, desde então, como efeito Meissner-Ochsenfeld. Registre-se que Meissner, nessa ocasião, se perguntava se essa supercondutividade se devia ao movimento de elétrons e íons, como acontecia nos condutores normais, ou se havia um outro tipo de portadores de carga. Ainda na década de 1930, duas teorias fenomenológicas foram desenvolvidas para explicar a supercondutividade: a termodinâmica, desenvolvida pelos físicos holandeses Cornelis Jacobus Gorter (1907-1980) e Hendrik Brugt Gerhard Casimir (1909-2000), em 1934; e a eletrodinâmica, elaborada pelos físicos alemães, os irmãos London, Fritz Wolfgang (1900-1954) e Heinz (1907-1970), em 1935, teorias essas que receberam, em 1950, um tratamento quanto-mecânico por parte dos físicos russos Vitaly Lazarevich Ginzburg (n. 1916; PNF, 2003) e Lev Davidovich Landau (1908-1968; PNF, 1962), tratamento esse que ficou conhecido como Teoria de Landau-Ginzburg, na qual os efeitos cooperativos dos elétrons são descritos por um campo escalar complexo.

A pergunta de Meissner começou a ser respondida, já em 1950, quando o físico alemão Herbert Fröhlich (1905-1992) e, independentemente, o físico norteamericano John Bardeen (1908-1991; PNF, 1956; 1972), propuseram uma teoria

${ }^{33}$ Progress in Theoretical Physics, v. 6, p. 1000, 1951.

Bassalo, J. M. F. 
para explicar aquele efeito. Eles afirmavam que o estado supercondutor era devido à interação entre os elétrons e a vibração (fônon) dos átomos no cristal, interação essa mais tarde conhecida como interação elétron-fônon que, em linhas gerais, significava que um elétron, ao deslocar-se em uma rede cristalina formada de íons positivos, era atraído por estes, provocando uma vibração local da rede. Em 1952, Fröhlich apresentou a ideia de que a interação elétron-fônon produzia uma atração entre elétrons, e que em muitos metais essa atração era ligeiramente superior à repulsão Coulombiana eletrônica. Em 1956, o físico norte-americano Leon Neil Cooper (n. 1930; PNF, 1972) mostrou que, se um elétron passar nas proximidades de um íon positivo de um cristal supercondutor, interagirá com o mesmo, provocará a vibração da rede cristalina e a consequente emissão de um fônon. Contudo, se um segundo elétron passar, subsequentemente, pelo mesmo íon, ele absorverá esse fônon, trocando momentos, e os dois elétrons caminharão juntos no menor estado de energia possível, constituindo o que ficou conhecido, desde então, como o par de Cooper. Note-se que, como esse par é formado de elétrons, que são férmions, porém com seus spins de valor $1 / 2$, em sentidos contrários, o spin resultante desse par é nulo e, portanto, ele passa a se comportar como um bóson. Em virtude disso, diz-se que, na formação desse par, há uma "bosonificação" de férmions.

É oportuno observar que o tipo de "bosonificação" referido acima foi primeiramente encontrado pelo físico russo Aleksandr Morkowitsch Polyakov (n.1945), em $1988^{34}$, ao mostrar que uma partícula bosônica tri-dimensional na presença de um campo de Chern-Simons adquiria um fator topológico que a fazia transformar-se em uma excitação fermiônica. Contudo, esse mecanismo de transmutação, que já havia sido demonstrado por Polyakov, teve um tratamento mais matemático, realizado pelo meu amigo, o físico brasileiro Luiz Carlos Lobato Botelho (n. 1960) em 1994 e $2000^{35}$.

As Teorias Fenomenológicas da Supercondutividade tratadas acima, indicavam que havia necessidade do desenvolvimento de uma Teoria Microscópica da Supercondutividade, uma vez que, naquelas teorias, ficava evidente que o fenômeno da supercondutividade se devia, de algum modo, à interação elétron-fônon. Desse modo, em 1957, Bardeen, Cooper e o físico norte-americano John Robert Schrieffer (n. 1931; PNF, 1972) desenvolveram a hoje célebre Teoria BCS, segun-

\footnotetext{
${ }^{34}$ Modern Physics Letters 3A, p. 325, 1988.

${ }^{35}$ Notas de Física CBPF-045/1994; International Journal of Modern Physics A, v. 15, p. $755,2000$.
} 
do a qual o estado supercondutor deve-se, essencialmente, a uma condensação de elétrons em pares de Cooper de momento linear comum e sendo representada por uma função de onda coerente única. Em 1958, o físico russo Nikolai Nikolaevich Bogoliubov (1900-1992) ${ }^{36}$ e, independentemente, o físico norte-americano Philip Warren Anderson (n. 1923; PNF, 1977) ${ }^{37}$, apresentaram também uma Teoria Microscópica da Supercondutividade. No entanto, enquanto Bogoliubov usou o conceito de quase-partícula, pois, para ele, a supercondutividade é descrita por uma mistura coerente de elétrons e dessas "partículas" (sobre essas "partículas" ou "buracos"), Anderson usou o conceito de localização: estado isolado quando um metal não-interagente é submetido a um grande potencial randômico. Ele havia desenvolvido este conceito ainda em $1958^{38}$, segundo o qual um único elétron poderia ser localizado sem considerar a interação Coulombiana. Observe-se que, em 1959, Bogoliubov ${ }^{39}$ e Anderson ${ }^{40}$ voltaram a tratar da supercondutividade com seus respectivos modelos.

Nessas teorias microscópicas da supercondutividade havia um fato intrigante, qual seja, uma quebra espontânea de simetria. Com efeito, no modelo de Bogoliubov, há uma mistura de elétrons e "quase-partículas" e, portanto, seus estados não são autoestados da carga elétrica. Por sua vez, na BCS, sua Hamiltoniana não é invariante pelo grupo de simetria gauge U(1) [que é o grupo que rege a interação eletromagnética: a "Quantum Electrodynamics" (QED), conforme dissemos acima], uma vez que ela envolve os pares de Cooper de carga - 2 e. Portanto, isso indicava que a supercondutividade é o resultado da quebra espontânea de simetria elétrica U(1). Esse resultado foi obtido, em $1960^{41}$, por Nambu, ao mostrar que as excitações coletivas são canceladas pela interação Coulombiana de longo alcance, ou seja, que os fônons sem massa são transformados em fônons massivos. Note-se que, neste artigo, ele usou os diagramas de Feynman. Esse

\footnotetext{
${ }^{36}$ Journal of Experimental and Theoretical Physics, Soviet Physics (JETP), v. 7, p. 41; Nuovo Cimento, v. 7, p. 794, 1958.

37

Physical Review, v. 112, p. 1900, 1977.

38

Physical Review, v. 109, p. 1492, 1958.

39

Uspekhi Fizika Nauk, v. 67, p. 549, 1959.

${ }^{40}$ Journal of Physics and Chemistry of Solids, v. 2, p. 26, 1959.

41

Physical Review, v. 117, p. 648, 1960.
}

Bassalo, J. M. F. 
mecanismo de transformar partículas sem massa em partículas massivas foi reencontrado, na década de 1960, em Partículas Elementares, conforme veremos mais adiante. Registre-se que, ainda em $1960^{42}$, o físico russo G. M. Eliashberg também usou aqueles diagramas no entendimento da supercondutividade.

De posse desse resultado, Nambu passou a estudar a quebra espontânea de simetria nas interações físicas. Assim, ainda em $1960^{43}$, ele mostrou haver essa quebra na simetria quiral, como, por exemplo, a simetria $\mathrm{U}_{5}=\exp \left(\mathrm{iA} \gamma_{5}\right.$ ), onde $\mathrm{A}$ é uma matriz e $\gamma_{5}=\gamma_{1} \gamma_{2} \gamma_{3} \gamma_{4}$, sendo $\gamma_{\mathrm{i}}(\mathrm{i}=1,2,3,4)$ a matriz de Dirac. Em $1961^{44}$ inspirado nos trabalhos de Nambu sobre quebra de simetria, o físico inglês Jeffrey Goldstone (n. 1933) desenvolveu o conceito de quebra espontânea de simetria em teorias Abelianas, do tipo U (1) e, com isso, mostrou que tal quebra é acompanhada de uma ou mais espécies de partículas sem massa, chamadas desde então de bósons de Nambu-Goldstone (bN-G) ou, simplesmente, bósons de Goldstone (bG). Logo em seguida, em $1962^{45}$, Goldstone, Salam e Weinberg mostraram que a existência desses bósons era um resultado geral em Teoria Quântica de Campos. Note-se que, em 1964, em trabalhos independentes, os físicos, o inglês Peter Ware Higgs (n. 1929), os belgas François Englert (n. 1932) e Robert Brout (n. 1928), e G. S. Guralnik, C. R. Hagen e o físico inglês Thomas Walter Bannerman Kibble (n. 1932) (de origem indiana), partindo da hipótese de que a simetria quebrada espontaneamente deveria ser local, e não global, como considerada até então, encontraram um mecanismo que tornava massivos os bN-G. Esse mecanismo ficou conhecido como mecanismo de Higgs, e o bóson de spin nulo correspondente de tal mecanismo, como bóson de Higgs (bH). Registre-se que, em $1966^{46}$, o físico brasileiro Jorge André Swieca (1936-1980) (meu professor na Universidade de São Paulo), David Kastler e Derek W. Robinson apresentaram uma demonstração axiomática do Teorema de Goldstone, com as seguintes hipóteses básicas: 1) invariância de Lorentz; 2) espaço de Hilbert com norma positiva definida, 3) simetria global.

\footnotetext{
${ }^{42}$ Soviet Physics - JETP, v. 11, p. 696, 1960.

43

Physical Revieis Letters, v. 4, p. 380, 1960.

44

Nuovo Cimento, v. 19, p. 154, 1961.

45

Physical Review, v. 127, p. 965, 1962.

${ }^{46}$ Communications in Mathematical Physics, v. 2, p. 108, 1966.
} 
Antes de prosseguirmos com os trabalhos dos Nobelistas, é oportuno falar um pouco mais sobre a quebra espontânea de simetria. As simetrias na Física das Partículas Elementares são realizadas em dois modos. O primeiro corresponde ao modo normal, ou modo das simetrias definidas por Wigner e Weyl, conforme vimos acima. Nesse caso, as partículas são descritas pelas representações irredutíveis dos grupos subjacentes $[\mathrm{SU}(\mathrm{N})]$, que explica os multipletos degenerados em energia ou em massa. O outro modo de realizar uma simetria é o de NambuGoldstone, também descrito acima. Neste modo, ao invés de as partículas serem distribuídas em multipletos, há o aparecimento de partículas com massa nula, os bN-G ou bG. Costuma-se dizer que a simetria de um sistema físico sofre uma quebra espontânea se o mais baixo estado desse sistema é não-invariante sob as operações daquela simetria. Diz-se também que, se um sistema físico não exibe todas as simetrias nas leis que o governam, então há quebra espontânea de simetria. Do ponto de vista formal, a quebra espontânea de simetria corresponde à situação em que a Lagrangiana é invariante pelo grupo de simetria, mas o vácuo não é invariante. Assim, para cada gerador da simetria quebrada espontaneamente aparece uma partícula de massa nula. Por isso, a quebra espontânea de simetria só ocorre no modo de Nambu-Goldstone ${ }^{47}$.

Parece que o primeiro exemplo de quebra espontânea de simetria foi apresentado pelo físico alemão Werner Karl Heisenberg (1901-1976; PNF, 1932), em $1928^{48}$, em seu célebre modelo do ferromagnetismo, segundo o qual o forte alinhamento dos spins (característica desse estado físico) decorria de uma energia de troca ("exchange") entre os spins dos elétrons vizinhos. Nesse modelo, ele usou os caracteres das representações do Grupo de Permutações e, também, a distribuição Gaussiana para incluir as flutuações nos níveis de energia eletrônicos. A quebra espontânea de simetria no ferromagnetismo decorre em virtude de a Hamiltoniana de Heisenberg ser invariante pelo grupo de rotações, mas o mesmo não ocorre com o estado de menor energia ou estado fundamental, quando todos os spins estão alinhados segundo alguma direção. Ora, como a direção do alinhamento é arbitrária, o vácuo é infinitamente degenerado. Desse modo, para estudarmos

${ }^{47}$ LEITE LOPES op. cit.; LEADER; PREDAZZI, op. cit.; MARCELO OTÁVIO CAMINHA GOMES Teoria Quântica dos Campos. EDUSP, 2002; Vicente Pleitez A Física das Partículas Elementares e os Prêmios Nobel de 2008. Boletim da Sociedade Brasileira de Física (018/2008). Aproveito a oportunidade para agradecer a esse físico brasileiro, nascido em El Salvador, em 1950, a leitura crítica deste artigo.

48

Zeitschrift fur Physik, v. 49, p. 619, 1928. 
as propriedades físicas do ferromagnetismo, temos de escolher um desses vácuos e, portanto, a simetria é quebrada. Registre-se que, nesse caso, os correspondentes bG, isto é, as oscilações de longo alcance são os magnons (os quanta das "ondas de spin"), que são produzidos pela oscilação da direção de polarização local, conforme foi mais tarde mostrado depois que o físico norte-americano Felix Bloch (1905-1983; PNF, 1953) descobriu esses quanta, em $1930^{49}$, e o aplicou ao ferromagnetismo (GOMES, op. cit.; PLEITEZ, op. cit.).

$\mathrm{Na}$ vida cotidiana, um exemplo de quebra espontânea de simetria é apresentado por Salam no livro Em Busca da Unificação ${ }^{50}$. Ele considera uma mesa circular na qual estão postos um guardanapo e um pão para cada convidado de uma maneira simétrica, aguardando a hora de comer; porém, para iniciar arefeição, cada convidado fica na dúvida quanto a qual guardanapo escolher, o da sua esquerda ou o da sua direita. No entanto, quando um dos convidados pega o seu guardanapo, a simetria é momentaneamente quebrada, porém, imediatamente, a relação de ordem com a simetria (representada pelo ato de escolha entre os estados simétricos possíveis) é estabelecida, pois os convidados agora sabem qual o guardanapo que devem escolher. Ainda nesse livro, Salam fala da quebra espontânea da simetria no magnetismo, que ocorre quando um ímã perde sua propriedade de se orientar para os pólos terrestres, quando ele é esquentado, acima de sua temperatura Curie $\left(\mathrm{T}_{\mathrm{C}}\right)$. Abaixo dessa temperatura, ele tem uma preferência de orientação no espaço indicada acima. Porém, quando ele se esquenta, perde essa preferência, ou seja, sua orientação passa a ser simétrica. No entanto, ao ser resfriado até abaixo de $\mathrm{T}_{\mathrm{C}}$, a sua orientação no espaço é recuperada, dizendo-se, então, que a simetria é espontaneamente quebrada ou que está "escondida". Segundo Pleitez (op. cit.), em vista disso, o correto seria chamar-se simetria escondida e não quebra espontânea de simetria.

Antes de tratarmos dos trabalhos dos Nobelistas Kobayashi e Maskawa, é oportuno registrar alguns aspectos de outros trabalhos de Nambu, fora do tema do PNF. O modelo de quarks proposto, independentemente, pelos físicos norteamericanos Murray Gell-Mann (n. 1929; PNF, 1969) e George Zweig (n. 1937) (de origem russa), em 1964, indicava que existiam partículas que eram formadas pela composição de três quarks, de sabores ("flavour"): $u p(u)$, down (d), e strange (s) [que aparecem como multipletos do $\mathrm{SU}(3)$ ], como, por exemplo, as resso-

${ }^{49}$ Leipziger Vorträge: Elektronen-Interferenzen, p. 167, 1930. 50 Gradiva, 1991. 
nâncias bosônicas: $\Delta^{++}$(uuu), $\Delta^{-}$(ddd) e $\Omega^{-}$(sss). Porém, como os quarks são férmions, essas três partículas violariam o Princípio da Exclusão de Pauli, formulado em 1925. Assim, para contornar essa dificuldade, o físico norte-americano Oscar Wallace Greenberg (n. 1932), em $1964^{51}$, propôs que cada quark era caracterizado por uma das três cores primárias do espectro luminoso: vermelho, azul e verde. Por sua vez, os antiquarks seriam caracterizados pelas cores complementares desse mesmo espectro: cyan, amarelo e magenta. (Registre-se que essas "cores", que nada têm a ver com as cores de nosso cotidiano, representam três novos graus de liberdade físicos.) Desse modo, teríamos [p.e.: $\Delta^{++}=[\mathrm{u}($ vermelho $), \mathrm{u}(\mathrm{azul})$, $\mathrm{u}(\mathrm{verde})]$. Essa proposta de Greenberg foi confirmada pelo físico koreano MooYoung Han (n.1934) e por Nambu, em $1965^{52}$. Registre-se que, ainda em 1964, trabalhos independentes de James Daniel Bjorken (n. 1934) e Glashow; D. Amati, H. Bacry, J. Nuyts e Jacques Prentki; Z. Maki e Yoshio Ohnuki (n. 1928); Lev Borisovich Okun (n. 1929) e W. Krolikowski; e Y. Yara estudaram a extensão do grupo $\mathrm{SU}(3)$ para o $\mathrm{SU}(4)$ e, com isso, aventaram a existência de um quarto quark, com sabor denominado de charme (c), por Bjorken e Glashow.

Uma outra importante contribuição de Nambu para a Física, fora do contexto do PNF, foi o seu trabalho na Teoria de Cordas. A ideia de corda ("string") é uma tentativa de generalizar o conceito de "partícula puntiforme", como eram consideradas (e ainda são) todas as partículas elementares até então conhecidas, em virtude do problema da "singularidade" (infinito) que decorre de sua dimensão zero (ponto). Em qualquer instante, a configuração de uma corda é uma curva que pode ser aberta ou fechada e, quando a mesma se move através do espaço-tempo, ela varre uma superfície conhecida como folha-mundo ("world-sheet"). No entanto, sendo classicamente a ação relativista para uma partícula livre proporcional ao comprimento invariante Lorentziano de sua linha-mundo ("world-line"), em 1970, em trabalhos independentes, Nambu ${ }^{53}$, Holger B. Nielsen ${ }^{54}$ e Leonard Susskind $^{55}$ e, em $1971^{56}$, o físico japonês T. Goto propuseram que a ação relativista

\footnotetext{
${ }^{51}$ Physical Review Letters, v. 13, p. 598, 1964.

Physical Review B, v. 139, p. 1006, 1965.

${ }^{53}$ Lectures at Copenhagen Summer Symposium

54 15th International Conference Physics, Kiev.

${ }^{55}$ Nuovo Cimento A, v. 69, p. 457, 1970.

${ }^{56}$ Progress in Theoretical Physics, v. 46, p. 1560, 1971.
} 
para uma corda deveria ser proporcional à área da folha-mundo, ação conhecida desde então como ação Nambu-Goto ${ }^{57}$.

Ainda sobre Nambu, é também oportuno registrar que ele orientou na Universidade de Chicago, em 1967, a Tese de Doutoramento de dois físicos brasileiros, Roland Köberle (n. 1938) (meu professor na Universidade de São Paulo) e Silvestre Ragusa (n. 1933), com os respectivos títulos: Proton Compton Scattering e Partially Conserved Axial-Vector Current Conditions on Strange Particles Photoproduction Amplitudes. O tema de espalhamento de partículas já havia sido objeto de pesquisa por parte de Nambu. Por exemplo, em $1957^{58}$, ele, o físico norte-americano Marvin Leonard Goldberger (n. 1922) e Oehme analisaram as relações de dispersão nas amplitudes de espalhamento. Em $1960^{59}$, ele estudou os fatores de forma de experiências envolvendo núcleons e interpretou alguns resultados como sendo devidos ao decaimento de uma partícula isoescalar em três píons. Em $1965^{60}$, Köberle, S. Fenster e Nambu estudaram os mésons vetoriais axiais e a estrutura hiperfina do hidrogênio $(\mathrm{H})$.

Agora, para finalizar este artigo, vejamos os trabalhos dos Nobelistas Kobayashi e Maskawa. Cabibbo, em 1963, ao examinar o decaimento de partículas estranhas (p. e. $K^{ \pm}$), explicou porque os processos com mudança de estranheza $(\Delta \mathrm{S})$, do tipo (na linguagem atual): $K^{-} \rightarrow \mu^{-}+\bar{v}_{\mu}$, acontecem com menor frequência do que aqueles em que não ocorre essa mudança, como, por exemplo, o decaimento: $\mu^{-} \rightarrow e^{-}+\bar{v}_{e}+v_{\mu}$. Para essa explicação, Cabibbo introduziu, no contexto da Álgebra de Correntes, um parâmetro conhecido como ângulo de Cabibbo $\theta_{\mathrm{C}}$, para explicar porque processos com $\Delta \mathrm{S}=1$ são suprimidos com relação àqueles com $\Delta S=0$. Registre-se que esse "ângulo" representa a relação entre as intensidades relativas desses dois decaimentos e significa a "projeção" de uma intensidade sobre a outra que se encontra inclinada daquele "ângulo". Na Teoria do Charme, desenvolvida em 1970, por Glashow, John Iliopoulos (n. 1940) e Luciano Maiani (n. 1941) (Teoria GIM), o $\theta_{\mathrm{C}}$ aparece naturalmente como um parâ-

\footnotetext{
${ }^{57}$ Para maiores detalhes sobre essa ação, ver: Michio Kaku, Introduction to Superstrings and M-Theory (Springer, 1999).

58

Annals of Physics (NY), v. 2, p. 226, 1957.

59

Physical Review, v. 106, p. 1366, 1960.

60

Physics Letters A, v. 19, p. 153, 1965.
} 
metro de uma rotação em um espaço abstrato bidimensional, o chamado "espaço dos sabores", e representado por uma matriz 2 x 2, a matriz de Cabibbo.

Depois que foi mostrada a quebra da simetria CP no sistema $\mathrm{K}^{0}-\overline{\mathrm{K}}^{0}$, em 1964, conforme vimos acima, verificou-se que a mesma é uma realidade física e, portanto, a sua procura em outros sistemas físicos passou a ser objeto de pesquisa. Essa procura foi encontrada por Kobayashi e Maskawa, em $1973^{61}$, ao mostrarem que interação eletrofraca, regida pelo grupo $\mathrm{SU}(2)_{\mathrm{L}} \otimes \mathrm{U}(1)_{\mathrm{Y}}$, viola a simetria $\mathrm{CP}$, desde que se considere, por exemplo, pares de três quarks, ou se aumente os campos de Higgs. Desse modo, a matriz 2 x 2 de Cabibbo deve ser substituída por uma matriz 3 x 3, agora denominada de matriz de Cabibbo-Kobayashi-Maskawa (CKM), que apresenta uma fase física depois de ser usada toda a arbitrariedade nas fases dos quarks. Lembre que, em Mecânica Quântica, apenas as fases relativas são observáveis. É oportuno registrar que o quinto quark, o bottom (b), foi descoberto em 1977, e o sexto quark, o top (t), em 1994. Registre-se que, em $1983^{62}$, Wolfestein apresentou uma conveniente parametrização da matriz KM (expansão dessa matriz em potências do parâmetro $\lambda$ ) para estudar as condições da violação da $\mathrm{CP}$.

A violação da CP, "direta e indireta", estudada pela CKM é a mesma da violação observada em 1964, por Christenson, Cronin, Fitch e Turlay (CCFT). No entanto, enquanto nesta, a observação decorre da mistura da matriz de massa, com os káons neutros transformando-se em antikáons (um quark substituído pelo seu antiquark) e vice-versa, porém com probabilidades diferentes, na CKM, a observação é realizada diretamente no decaimento daquelas partículas. Na verdade, Kobayashi e Maskawa estudaram o mecanismo de violação de CP que leva seu nome, mecanismo esse que se ajusta bem com os dados, tanto da violação direta e indireta nos káons como na direta nos mésons B, foi feito por outros autores. Ambas, contudo, são parametrizadas. No entanto, o parâmetro $(\lambda)$ da CKM é cerca de mil vezes menor do que o da CCFT, daí a dificuldade de ele ser medido. Uma primeira medida foi realizada, em 1993, por Turlay e mais 28 físicos do Fermilab E731 Collaboration $^{63}$ e, independentemente, por G. D. Barr e mais 48 físicos do CERN NA31 Collaboration ${ }^{64}$, ao observarem a violação de CP no decaimento do sistema

\footnotetext{
61

Progress in Theoretical Physics, v. 49, p. 652, 1973.

62

Physical Review Letters, v. 51, p. 1945, 1983.

63

Physical Review Letters, v. 70, p. 1203, 1993.

64

Physics Letters B, v. 317, p. 233, 1993.
}

Bassalo, J. M. F. 
káon neutro $\left(\mathrm{K}^{0}-\overline{\mathrm{K}}^{0}\right)$. Essa prova experimental, no entanto, foi questionada. Uma nova prova experimental foi pesquisada, em $1997^{65}$ e $1998^{66}$, pelos físicos brasileiros Sandra Amato (n. 1963), João Carlos Costa dos Anjos (n. 1944), Ignácio Alfonso de Bediaga e Hickman (n. 1954), Hendly da Silva Carvalho (n. 1966), Carla Gobel (n. 1969), João Ramos Torres de Mello Neto (n. 1960), Jussara Marques de Miranda (n. 1962), Alberto Correa dos Reis (n. 1958) e Alberto Franco de Sá Santoro (n. 1941) e mais, respectivamente, 61 e 64 físicos da Fermilab E791 Collaboration, ao estudarem o decaimento dos mésons charmosos neutros $\mathbf{D}^{\mathbf{0}}$ (esses mésons possuem em sua estrutura o quark c). Por fim, uma outra violação de $\mathrm{CP}$ direta dos káons foi estudada, em $1999^{67}$ em uma experiência realizada no CERN (NA48 Collaboration), com a participação de Turlay e mais 153 físicos, na qual examinaram o decaimento: $K^{0} \rightarrow \pi^{+}+\pi^{-}$. Em 2001, B. Aubert e mais 619 físicos do Stanford Linear Accelerator Center (SLAC), nos Estados Unidos (BaBar Collaboration) ${ }^{68}$ e, independentemente, K. Abe e mais 251 físicos do High Energy Accelerator Research Organization (“Enerug Kasokuki Kenky Kik” - KEK), no Japão (Belle Collaboration) ${ }^{69}$ realizaram experiências nas quais mostraram que a violação da CP também acontece no sistema de mésons bonitos $\left(B^{0}-\bar{B}^{0}\right)$ (esses mésons têm, em sua estrutura, o quark b). Registre-se que, nessas duas últimas experiências, a violação foi confirmada pela medida do parâmetro sen $(2 \beta) \neq 0$, que expressa a assimetria matéria-antimatéria.

A violação "direta" da simetria $\mathrm{CP}$ foi também objeto de estudo teórico envolvendo os decaimentos de mésons charmosos e bonitos. Por exemplo, em $1993^{70}$ por João M. Soares e Wolfenstein discutiram a violação da CP na produção do méson charmoso B. Em 1994 $4^{71}$, M. Gronau, D. London e J. L. Rosner estudaram o decaimento: $\mathrm{B}^{ \pm} \rightarrow \mathrm{K}^{ \pm}+\pi^{0}$. Em $1996^{72}$, M. Beneke, G. Buchalla e I. Duni-

65

Physics Letters B, v. 403, p. 377, 1997.

66

Physics Letters B, v. 421, p. 405, 1998.

67

Physics Letters B, v. 465, p. 335, 1999.

68

Physical Review Letters, v. 87, p. 091801, 2001.

69

Physical Review Letters, v. 87, p. 091802, 2001.

70

Physical Review D, v. 47, p. 1021, 1993.

${ }^{71}$ Physical Review Letters, v. 73, p. 21, 1994. 72

Physical Review D, v. 54, p. 4419, 1996. 
etz investigaram a violação no sistema $\mathrm{B}^{0}-\overline{\mathrm{B}}^{0}$. Em $1997^{73}$, B. Blok, Gronau e Rosner; e em $1999^{74}$, R. Fleischer analisaram os decaimentos: $\mathrm{B}^{0} \rightarrow \mathrm{K}^{+}+\mathrm{K}^{-} \mathrm{e}$ $\mathrm{B}_{\mathrm{S}}^{0} \rightarrow \pi^{+}+\pi^{-}$. Em $1998^{75}$, M. Neubert e Rosner; em $1999^{76}$, Xiao-Gang He, WeiShu Hou e Kwei-Chou Yang; e em $2000^{77}$, Gronau e Rosner estudaram os decaimentos: $B^{0} \rightarrow \pi^{+}+\pi^{-}$e $\mathrm{B}^{0} \rightarrow \mathrm{K}^{*+}+\pi^{-}$. Em 1999, Fleischer ${ }^{78}$ e, independentemente, D. Pirjol ${ }^{79}$, e em 2000, Fleischer ${ }^{80}$ estudaram a violação da CP nos decaimentos: $\mathrm{B}_{\mathrm{S}}^{0} \rightarrow \mathrm{K}^{+}+\mathrm{K}^{-}$e $B^{0} \rightarrow \pi^{+}+\pi^{-}$. Registre-se que, em $1995^{81}$, C. P. Yuan estudou a violação da $\mathrm{CP}$ no decaimento do quark $\mathrm{t}$.

Na conclusão deste artigo, é oportuno tecer dois comentários. O primeiro, relaciona-se com as duas previsões da violação da $\mathrm{CP}: 1)$ a assimetria matériaantimatéria (bárion-antibárion) observada no Universo. Em $1967^{82}$, o físico russo Andrey Dmitriyevich Sakharov (1921-1989; PNPaz, 1975) sugeriu que aquela assimetria poderia ser associada à violação da CP, como a observada, em 1964, no sistema $\mathrm{K}^{0}-\overline{\mathrm{K}}^{0}$, e tratada neste artigo. Com efeito, segundo a Teoria do BigBang, se a CP fosse preservada, essa explosão deveria produzir quantidades iguais de matéria e de antimatéria e que se cancelariam, produzindo um mar de fótons (em virtude do processo físico conhecido como annilation), no Universo sem matéria. Como o Universo está cheio de matéria, então, segundo Sakharov, houve

${ }^{73}$ Physical Review Letters, v. 78; 79; p. 3999; 1167, 1997.

${ }^{74}$ European Physics Journal C, v. 6, p. 451, 1999.

${ }^{75}$ Physics Letters B, v. 441, p. 403, 1998; Physical Review Letters, v. 81, p. 5076, 1998.

${ }^{76}$ Physical Review Letters, v. 83, p. 1100, 1999.

${ }^{77}$ Physical Review D, v. 61, p. 073008, 2000.

${ }^{78}$ Physics Letters B, v. 459, p. 306, 1999.

${ }^{79}$ Physical Review D, v. 60, p. 054020, 1999.

${ }^{80}$ European Physics Journal C, v. 16, p. 87, 2000.

${ }^{81}$ Modern Physics Letters A, v. 10, p. 627, 1995.

${ }^{82}$ Pis'ma Zhurnal Eksperimental'noi I Teoreticheskoi Fiziki, v. 5, p. 32, 1997; JEPT Letters, v. 5, p. 24, 1997. 
uma violação da CP imediatamente depois do Big-Bang; 2) a existência de momento de dipolo elétrico (MDE) para o nêutron e da ordem de $10^{-18}$ e.m.

Segundo Pleitez ${ }^{83}$, a procura pela existência do MDE é bem mais antiga. Com efeito, em $1957^{84}$, Landau colocou esse problema no contexto da violação da paridade (P). Nessa ocasião, ele afirmou que se $\mathrm{P}$ for violada então um estado quântico não degenerado pode apresentar um MDE. Era a conservação de $\mathrm{P}$ que impedia isso. Quando foi observada a violação de P, ainda em 1957, acreditou-se que seria a conservação de $\mathrm{CP}$ a que impediria o MDE naquele tipo de estado. Com a observação da violação de CP, em 1964, ficou claro que o nêutron e outras partículas elementares ou mesmo núcleos podem apresentar um MDE. Só que, no mecanismo de $\mathrm{KM}$, ele é muito pequeno, menor que $10^{-32} \mathrm{e}-\mathrm{cm}$. Porém, o limite experimental está na ordem dos $10^{-26} \mathrm{e}-\mathrm{cm}$. Assim, se ele fosse observado, por exemplo, com $10^{-27} \mathrm{e}-\mathrm{cm}$, seria uma prova de que existem novas fontes de violação $\mathrm{CP}$ diferente da do mecanismo de KM.

Mais tarde, quando o físico holandês Gerardus't Hooft (n. 1946; PNF, 1999), em $1976^{85}$, descobriu o instanton (como solução da TYMS), ele observou que isso levaria à violação de CP pela Lagrangiana da QCD. Logo depois dessa descoberta, em 1977, o físico e matemático norte-americano Edward Witten (n. $1951)^{86}$ e, independentemente, Roman Jackiw, C. Nohl e C. Rebbi ${ }^{87}$, observaram que, no caso daquela violação da $\mathrm{CP}$, o $\mathrm{MDE}$ do nêutron teria uma contribuição dessa Lagrangiana. É oportuno registrar que a QCD preserva a simetria CP. Até o momento (dezembro de 2008), o MDE não foi observado porque esse valor previsto é muito ${ }_{88}$ do que a capacidade experimental atual para medi-lo, conforme falamos acima $^{88}$.

O segundo comentário tem relação com o limite de validade do Modelo Padrão, que é o modelo atual que explica as interações físicas (forte e eletrofraca) das partículas elementares, em decorrência da violação da CP. Enquanto a intera-

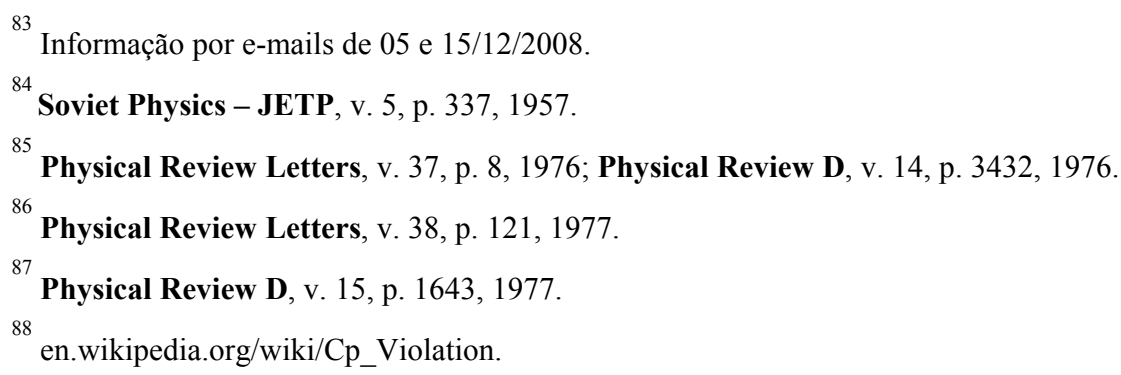


ção eletrofraca (TWS) viola a CP, conforme vimos neste trabalho, a interação forte (QCD) não a viola, como afirmamos acima. A violação da CP na TWS decorre do fato de que os seus campos de gauge (YMS) se acoplam a correntes quirais construídos de campos fermiônicos e, no caso do Modelo Padrão, pela existência de 6 quarks. Na verdade, o que importa para termos violação de CP é o de haver uma ou mais fases físicas. Por seu lado, a preservação da $\mathrm{CP}$ na $\mathrm{QCD}$, é consequência de os glúons se acoplarem a correntes vetoriais, muito embora existam termos naturais na Lagrangiana da QCD que permitam a quebra espontânea da CP. Esse problema, hoje conhecido como SCPP (“Strong CP Problem”), é que está colocando em xeque até que ponto vale o Modelo Padrão. É oportuno salientar que existem tentativas teóricas que estão procurando resolver esse problema, com o envolvimento ou não de novas partículas escalares conhecidas como axions. A primeira proposta foi apresentada em $1977^{89}$, por R. D. Peccei e H. R. Quinn. A segunda, envolvendo dimensões espaço-temporais duplas no tempo, foi apresentada em $1998^{90}$, por I. Bars, C. Deliduman e O. Andreev ${ }^{91}$.

${ }^{89}$ Physical Review Letters, v. 38, p. 1440, 1977; Physical Review D, v. 16, p. 179, 1977.

90

Physical Review D, v. 28, p. 066004, 1988.

91

Para maiores detalhes, ver: en.wikipedia.org/wiki/Cp_Violation.

Bassalo, J. M. F. 